\title{
Plasma glial fibrillary acidic protein is raised in progranulin-associated frontotemporal dementia
}

\author{
Carolin Heller (D) ,' Martha S Foiani (D) ,' Katrina Moore (D) ,' Rhian Convery (D) , \\ Martina Bocchetta (D) , ${ }^{2}$ Mollie Neason (D) ,' David M Cash, 2,3 David Thomas (D) , 4 \\ Caroline V Greaves (D) , ${ }^{2}$ Ione OC Woollacott (D) ,' Rachelle Shafei (D) , \\ John C Van Swieten (1) , ${ }^{5}$ Fermin Moreno, ${ }^{6}$ Raquel Sanchez-Valle, ${ }^{7}$ \\ Barbara Borroni (D) , ${ }^{8}$ Robert Laforce Jr, ${ }^{9}$ Mario Masellis, ${ }^{10}$ Maria Carmela Tartaglia, ${ }^{11}$ \\ Caroline Graff, ${ }^{12}$ Daniela Galimberti, ${ }^{13,14}$ James B Rowe (D) , ${ }^{15}$ Elizabeth Finger (D) , 16 \\ Matthis Synofzik, ${ }^{17,18}$ Rik Vandenberghe, ${ }^{19}$ Alexandre de Mendonca, ${ }^{20}$ \\ Fabrizio Tagliavini, ${ }^{21}$ Isabel Santana, ${ }^{22}$ Simon Ducharme, ${ }^{23}$ Christopher R Butler, ${ }^{24}$ \\ Alex Gerhard, ${ }^{25}$ Johannes Levin, ${ }^{26,27,28}$ Adrian Danek, ${ }^{27}$ Giovanni Frisoni, $^{29}$ \\ Sandro Sorbi, ${ }^{30}$ Markus Otto (D) , ${ }^{31}$ Amanda J Heslegrave, ${ }^{1}$ Henrik Zetterberg, ${ }^{1,32}$ \\ Jonathan D Rohrer (D) , ${ }^{2}$ on behalf of GENFI
}

- Additional material is

published online only. To view, please visit the journal online (http://dx.doi.org/10.1136/ jnnp-2019-321954).

For numbered affiliations see end of article.

Correspondence to Dr Jonathan D Rohrer, Dementia Research Centre, UCL Institute of Neurology, London, UK; j. rohrer@ucl.ac.uk

Received 28 August 2019 Revised 20 November 2019 Accepted 2 December 2019 Published Online First 14 January 2020

\section{Check for updates}

(C) Author(s) (or their employer(s)) 2020. No commercial re-use. See rights and permissions. Published by BMJ.

To cite: Heller C, Foiani MS, Moore K, et al. J Neurol Neurosurg Psychiatry 2020;91:263-270.

\section{ABSTRACT}

Background There are few validated fluid biomarkers in frontotemporal dementia (FTD). Glial fibrillary acidic protein (GFAP) is a measure of astrogliosis, a known pathological process of FTD, but has yet to be explored as potential biomarker.

Methods Plasma GFAP and neurofilament light chain (NfL) concentration were measured in 469 individuals enrolled in the Genetic FTD Initiative: 114 C9orf72 expansion carriers (74 presymptomatic, 40 symptomatic), 119 GRN mutation carriers (88 presymptomatic, 31 symptomatic), 53 MAPT mutation carriers (34 presymptomatic, 19 symptomatic) and 183 non-carrier controls. Biomarker measures were compared between groups using linear regression models adjusted for age and sex with family membership included as random effect. Participants underwent standardised clinical assessments including the MiniMental State Examination (MMSE), Frontotemporal Lobar Degeneration-Clinical Dementia Rating scale and MRI. Spearman's correlation coefficient was used to investigate the relationship of plasma GFAP to clinical and imaging measures.

Results Plasma GFAP concentration was significantly increased in symptomatic GRN mutation carriers (adjusted mean difference from controls $192.3 \mathrm{pg} / \mathrm{mL}$, 95\% Cl 126.5 to 445.6 ), but not in those with C9orf72 expansions (9.0, -61.3 to 54.6), MAPT mutations (12.7, -33.3 to 90.4$)$ or the presymptomatic groups. GFAP concentration was significantly positively correlated with age in both controls and the majority of the disease groups, as well as with NfL concentration. In the presymptomatic period, higher GFAP concentrations were correlated with a lower cognitive score (MMSE) and lower brain volume, while in the symptomatic period, higher concentrations were associated with faster rates of atrophy in the temporal lobe.

Conclusions Raised GFAP concentrations appear to be unique to $G R N$-related FTD, with levels potentially increasing just prior to symptom onset, suggesting that
GFAP may be an important marker of proximity to onset, and helpful for forthcoming therapeutic prevention trials.

\section{INTRODUCTION}

Frontotemporal dementia (FTD) is a progressive neurodegenerative condition with around a third of cases caused by an autosomal dominant gene mutation in progranulin $(G R N)$, chromosome 9 open reading frame 72 (C9orf72) or microtubuleassociated protein tau $(M A P T) .{ }^{1}$ As clinical trials in genetic FTD are fast approaching, robust biomarkers that allow accurate measurement of disease onset and progression are becoming increasingly important. In particular, many trials will focus on the presymptomatic stage of disease where neuropathological alterations are already present ${ }^{2}$ and yet few biomarkers have been shown to be abnormal in this phase. ${ }^{3-5}$

Cerebrospinal fluid (CSF) or plasma/serum progranulin levels in GRN mutation carriers ${ }^{4} 6$ and CSF (poly)GP dipeptide repeat concentrations in C9orf72 expansion carriers ${ }^{58}$ are markers of specific protein abnormalities in genetic FTD, but both are abnormal from early in the presymptomatic period (and potentially from birth). In contrast, neurofilament light chain (NfL) is a marker of neuronal death and axonal degeneration (measurable in $\operatorname{CSF}^{3910}$ as well as both plasma ${ }^{11}$ and serum $^{12}{ }^{13}$ ) that is not specific to FTD ${ }^{14}$ and has only been shown to be abnormal in the very late presymptomatic period prior to conversion to the symptomatic phase. ${ }^{3}$ Glial fibrillary acidic protein (GFAP) is a marker of astrogliosis, the abnormal proliferation of astrocytes due to neuronal damage $^{15}$ and has previously been shown to be increased in frontal cortical tissue in FTD,${ }^{16}$ and raised in both the CSF and serum of patients with symptomatic FTD. ${ }^{17-19}$ However, it has yet to be 
Table 1 Demographic, cognitive and biomarker data from study participants

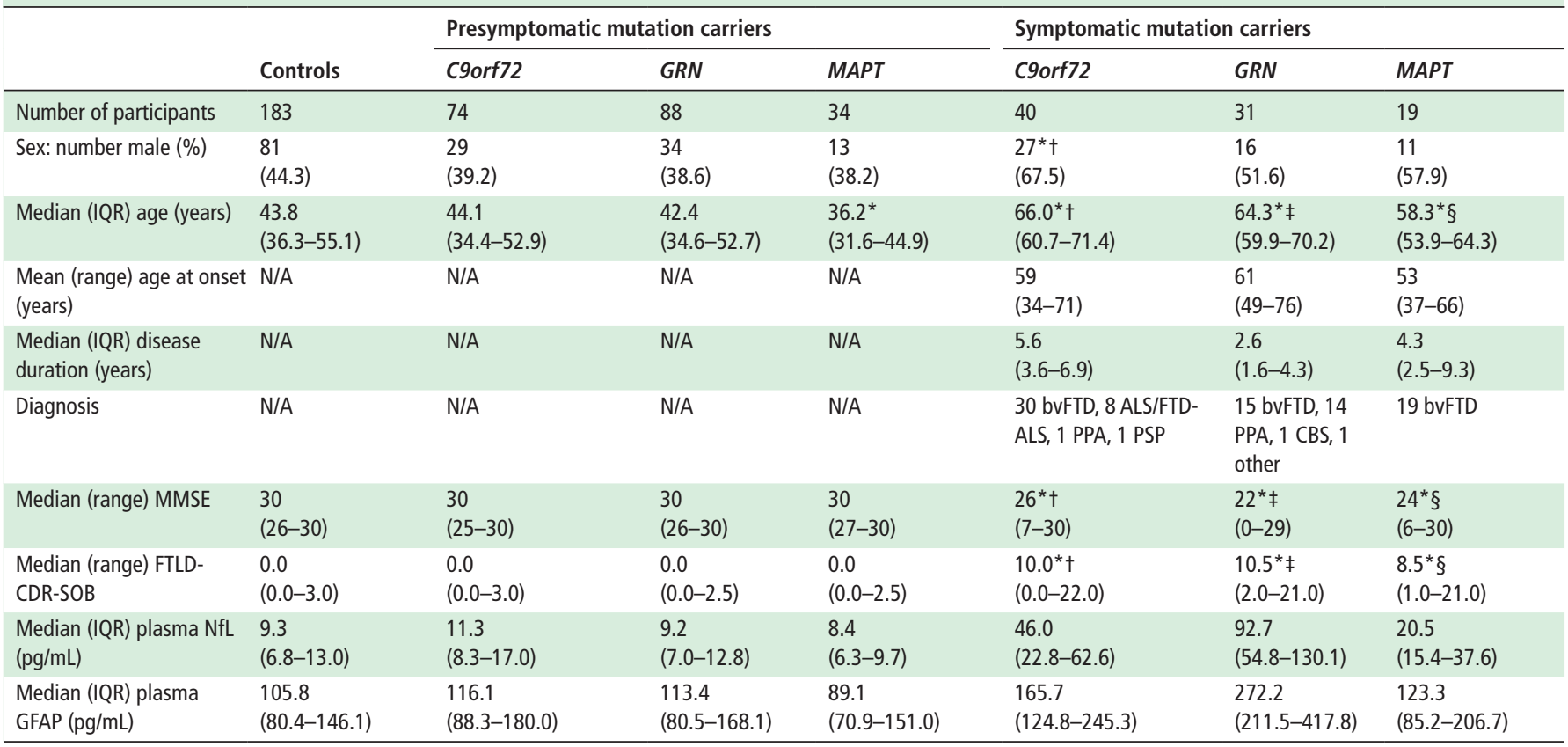

For sex, age, MMSE and FTLD-CDR-SOB, significant differences are shown at $\mathrm{p}<0.05$ :

${ }^{*}$ Compared with controls.

†In C9orf72 group between symptomatic and presymptomatic carriers.

‡ln GRN group between symptomatic and presymptomatic carriers.

§In MAPT group between symptomatic and presymptomatic carriers.

ALS, amyotrophic lateral sclerosis; bvFTD, behavioural varaint frontotemporal dementia; CBS, corticobasal syndrome; FTLD-CDR-SOB, Frontotemporal Lobar Degeneration-Clinical Dementia Rating scale-Sum Of Boxes; GFAP, glial fibrillary acidic protein; MMSE, Mini-Mental State Examination; N/A, not applicable; NfL, neurofilament light chain; PPA, primary progressive aphasia; PSP, progressive supranuclear palsy.

explored using ultrasensitive blood-based assays in genetic FTD mutation carriers.

In this study, we aimed to investigate within the Genetic FTD Initiative (GENFI) cohort whether plasma GFAP was abnormal in each of the different genetic FTD groups during the symptomatic period, and whether we could detect any presymptomatic changes. We also aimed to explore the relationship of GFAP with plasma NfL, cognitive, and neuroimaging measures.

\section{METHODS}

\section{Participants}

Participants were recruited from GENFI, a natural history study of genetic FTD involving 23 research centres across Europe and Canada (www.genfi.org.uk) ${ }^{2}$ involving symptomatic carriers of mutations in GRN, MAPT or C9orf72, and those at risk of carrying a mutation because a first-degree relative was a known symptomatic carrier. Four hundred sixty-nine consecutively recruited individuals from the GENFI study were included: 114 C9orf72 expansion carriers (74 presymptomatic, 40 symptomatic), 119 GRN mutation carriers (88 presymptomatic, 31 symptomatic), 53 MAPT mutation carriers (34 presymptomatic, 19 symptomatic) and 183 non-carriers who acted as a control group. Demographic information is shown in table 1: age and sex differed significantly between groups. All people in the study underwent a clinical assessment consisting of a medical history with the participant and informant, and physical examination, with symptomatic status diagnosed by a clinician who was an expert in the FTD field ${ }^{20-24}$ (specific diagnoses are shown in table 1). All participants underwent a standardised examination including the Mini-Mental State Examination (MMSE) and the Frontotemporal Lobar Degeneration-Clinical Dementia Rating scale (FTLD-CDR). ${ }^{25}$ Participants also performed three-dimensional
T1-weighted MRI of the brain: 432 scans were available for cross-sectional analysis, of which a subgroup of 243 participants had a follow-up scan (on the same scanner) for analysis (mean (SD) interval $1.12(0.29)$ years between baseline and follow-up). Volumetric measures of whole brain and cortical regions were calculated using a previously described method that uses the geodesic information flow (GIF) algorithm, which is based on atlas propagation and label fusion (online supplementary table 1). ${ }^{326}$ An annualised longitudinal rate of atrophy was found by calculating the difference in each specific measure between the baseline and longitudinal scan and expressing it as a percentage of the baseline volume over 1 year (online supplementary table 2).

\section{Measurement of plasma markers}

Plasma was collected, processed and stored in aliquots at $-80^{\circ} \mathrm{C}$ according to standardised procedures. Samples were measured using the multiplex Neurology 4-Plex A kit (102153, Quanterix, Lexington, USA) on the SIMOA HD-1 Analyzer following manufacturer's instructions. The lower limit of detection of the assay for GFAP and NfL were 0.221 and $0.104 \mathrm{pg} / \mathrm{mL}$, respectively. Measurements were carried out at the same study site on consecutive days and the operator was blinded to all clinical information, including genetic status. To keep sample processing and plating consistent, plasma samples were thawed at room temperature for 2 hours and subsequently centrifuged at $10000 \mathrm{~g}$ for $5 \mathrm{~min}$; $150 \mu \mathrm{L}$ samples were aliquoted in a 96-well plate (Quanterix) and frozen at $-80^{\circ} \mathrm{C}$ until analysis. Quality control samples had a mean intra-assay and interassay coefficient of variation of $<10 \%$.

\section{Statistical analysis}

Fisher's exact test was used to compare sex frequencies between groups. Distributions for demographic and biomarker data 
were investigated graphically using histograms and quantilequantile plots and tested for normality using the Shapiro-Wilk test. As demographic data did not follow a normal distribution, group differences for age at sample collection and FTLD-CDRSum Of Boxes were compared using the Kruskal-Wallis test. A linear regression adjusting for age was used to compare MMSE scores between groups. The primary analysis in the study was to investigate whether there were any differences in plasma GFAP concentration from controls in the different genetic mutation groups both symptomatically and presymptomatically, as well as between genetic groups. As biomarker values were not normally distributed, group means were compared by performing a linear mixed regression model with $95 \%$ bias-corrected bootstrapped CIs with 2000 repetitions in STATA (V.14; StataCorp, College Station, Texas, USA), adjusting for age and sex with family membership included as a random effect. Diagnostic performance of GFAP was assessed by areas under the curve (AUC) obtained by receiver operating characteristic (ROC) analyses, with optimal cut-off levels at the highest Youden's index (sensitivity+specificity-1) using GraphPad Prism (V.6; GraphPad Software, San Diego, California, USA). In order to investigate the relationship of GFAP concentration to demographic, cognitive and imaging measures as well as NfL concentrations, Spearman's correlation coefficient was used.

\section{RESULTS}

\section{Plasma GFAP concentration}

Plasma GFAP concentration was significantly higher in the symptomatic GRN mutation carriers compared with controls (adjusted mean difference $192.3 \mathrm{pg} / \mathrm{mL}$, 95\% CI 126.5 to 445.6), but not in either the symptomatic C9orf72 (9.0, -61.3 to 54.6) or MAPT (12.7, -33.3 to 90.4$)$ groups (figure 1 , tables 1 and 2). Within the symptomatic groups, concentrations in GRN were significantly higher than both C9orf72 (183.3, 106.1 to 427.2) and MAPT (179.6, 99.8 to 348.1) mutation carriers.

A ROC curve analysis measuring the ability of GFAP to distinguish symptomatic GRN mutation carriers from controls showed a sensitivity of $90.3 \%$ and specificity of $82.0 \%$ with a cut-off point of $163.2 \mathrm{pg} / \mathrm{mL}$ and an AUC of 0.90 . For distinguishing symptomatic GRN mutation carriers from C9orf72 mutation carriers there was a sensitivity of $71.0 \%$ and specificity of $70.0 \%$ with a cut-off point of $226.2 \mathrm{pg} / \mathrm{mL}$ and an AUC of 0.74 , while for distinguishing symptomatic GRN mutation carriers from MAPT mutation carriers there was a sensitivity of $79.0 \%$ and specificity of $77.4 \%$ with a cut-off point of $209.1 \mathrm{pg} / \mathrm{mL}$ and an AUC of 0.80 (online supplementary figure 1).

In the presymptomatic groups, concentrations were not significantly increased in any of the groups compared with controls: GRN (14.2, -2.4 to 38.3), C9orf72 (21.1, -18.8 to 66.5$)$, MAPT $(-7.0,-61.8$ to 7.8$)$ (figure 1 , tables 1 and 2 ). There were also no differences across the presymptomatic groups.

Comparing symptomatic and presymptomatic carriers, a significantly higher concentration was also seen in the symptomatic versus the presymptomatic GRN mutation carriers (178.1, 114.3 to 365.2 ), but not in the other groups (figure 1 , tables 1 and 2).

\section{Correlation with age}

GFAP concentration was significantly correlated with age at sample collection in controls $(\mathrm{r}=0.55, \mathrm{p}<0.001)$, presymptomatic mutation carriers (all groups combined: $\mathrm{r}=0.53, \mathrm{p}<0.001$; GRN: $\mathrm{r}=0.58, \mathrm{p}<0.001 ;$ C9orf72: $\mathrm{r}=0.50, \mathrm{p}<0.001 ;$ MAPT: $\mathrm{r}=0.36, \mathrm{p}=0.036)$ and symptomatic mutation carriers for all
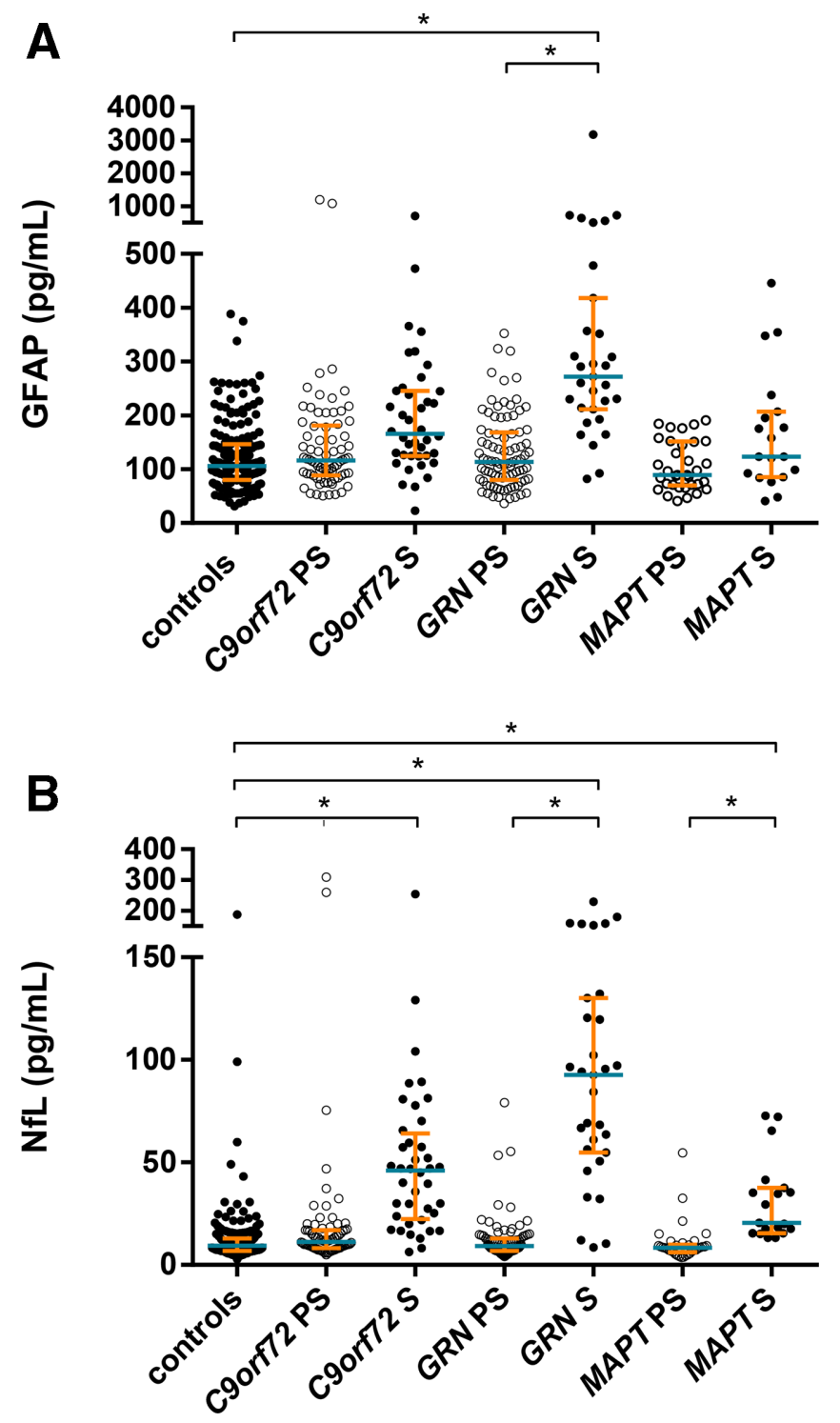

Figure 1 (A) Plasma glial fibrillary acidic protein (GFAP) and (B) plasma neurofilament light chain ( $\mathrm{NfL}$ ) concentrations $(\mathrm{pg} / \mathrm{mL}$ ) in control, presymptomatic and symptomatic frontotemporal dementia mutation carriers for each gene: C9orf72, GRN and MAPT. Median designated by blue line; IQRs indicated by orange error bars. * Significant differencesonly differences from controls and within the same genetic group are shown on the graph. Note that 29 data points fall outside the upper or lower limit using the IQR method, with factor $k=1.5$ (ie, outside the upper limit Q3+1.5×IQR or lower limit Q1-1.5xIQR): excluding these outliers does not change the significance of the results.

genetic groups together $(\mathrm{r}=0.38, \mathrm{p}<0.001)$ and the $G R N$ group alone $(\mathrm{r}=0.65, \mathrm{p}<0.001)$ (figure 2$)$. No significant correlation was seen for the symptomatic C9orf72 $(\mathrm{r}=0.27, \mathrm{p}=0.088)$ or MAPT mutation carriers $(\mathrm{r}=0.00, \mathrm{p}=0.989)$.

\section{Correlation with plasma NfL}

Plasma NfL was increased in all three symptomatic groups compared with controls (figure 1, tables 1 and 3): GRN mutation carriers (adjusted mean difference $70.5 \mathrm{pg} / \mathrm{mL}, 95 \%$ CI 51.6 to 92.6), C9orf72 mutation carriers $(29.7,18.7$ to 47.5$)$ and MAPT mutation carriers $(12.6,3.3$ to 26.1$)$. Within the symptomatic groups, concentrations in GRN were significantly higher than both C9orf72 $(40.7,16.5$ to 62.2$)$ and MAPT (57.9, 36.8 


\begin{tabular}{|c|c|c|c|c|c|c|}
\hline GFAP & C9orf72 PS & C9orf72 S & GRN PS & GRN S & MAPT PS & MAPT S \\
\hline Controls & $\begin{array}{l}21.1 \\
(-18.8 \text { to } 66.5)\end{array}$ & $\begin{array}{l}9.0 \\
(-61.3 \text { to } 54.6)\end{array}$ & $\begin{array}{l}14.2 \\
(-2.4 \text { to } 38.3)\end{array}$ & $\begin{array}{l}192.3^{* *} \\
(126.5 \text { to } 445.6)\end{array}$ & $\begin{array}{l}-7.0 \\
(-61.8 \text { to } 7.8)\end{array}$ & $\begin{array}{l}12.7 \\
(-33.3 \text { to } 90.4)\end{array}$ \\
\hline C9orf72 PS & & $\begin{array}{l}12.1 \\
(-64.1 \text { to } 85.1)\end{array}$ & $\begin{array}{l}-6.9 \\
(-51.0 \text { to } 43.0)\end{array}$ & $\begin{array}{l}171.2^{* *} \\
(88.5 \text { to } 433.7)\end{array}$ & $\begin{array}{l}-28.1 \\
(-83.3 \text { to } 8.0)\end{array}$ & $\begin{array}{l}8.4 \\
(-87.5 \text { to } 74.5)\end{array}$ \\
\hline C9orf72 S & & & $\begin{array}{l}-5.2 \\
(-79.4 \text { to } 39.4)\end{array}$ & $\begin{array}{l}183.3^{* *} \\
\text { (106.1 to } 427.2 \text { ) }\end{array}$ & $\begin{array}{l}-16.0 \\
(-84.3 \text { to } 32.9)\end{array}$ & $\begin{array}{l}-3.7 \\
(-104.5 \text { to } 56.1)\end{array}$ \\
\hline GRN PS & & & & $\begin{array}{l}178.1 * * \\
\text { (114.3 to } 365.2)\end{array}$ & $\begin{array}{l}-21.2 \\
(-78.3 \text { to } 1.5)\end{array}$ & $\begin{array}{l}1.5 \\
(-75.4 \text { to } 49.1)\end{array}$ \\
\hline GRNS & & & & & $\begin{array}{l}-199.3^{* *} \\
(-439.2 \text { to }-124.9)\end{array}$ & $\begin{array}{l}-179.6^{* *} \\
(-348.1 \text { to }-99.8)\end{array}$ \\
\hline MAPT PS & & & & & & $\begin{array}{l}19.6 \\
(-30.8 \text { to } 114.4)\end{array}$ \\
\hline
\end{tabular}

Significant differences in bold: ${ }^{*} \mathrm{p}<0.01$.

GFAP, glial fibrillary acidic protein; PS, presymptomatic; S, symptomatic.

to 81.5) mutation carriers, and C9orf72 mutation carriers were higher than MAPT mutation carriers (17.2, 2.9 to 35.7). Concentration was also increased in presymptomatic C9orf72 mutation carriers compared with controls $(9.0,1.3$ to 26.8), but not in the GRN or MAPT presymptomatic groups (figure 1, tables 1 and 3 ). Comparing symptomatic and presymptomatic carriers, a significantly higher concentration was also seen in the symptomatic versus the presymptomatic mutation carriers in each of the groups (figure 1, tables 1 and 3): GRN mutation carriers (70.5, 52.5 to 92.2 ), C9orf72 mutation carriers $(20.7,3.2$ to 36.1$)$ and MAPT mutation carriers $(11.7,0.8$ to 23.4$)$.

Plasma GFAP and NfL concentrations were significantly correlated in controls $(\mathrm{r}=0.66, \mathrm{p}<0.001)$, presymptomatic mutation carriers $(G R N: \mathrm{r}=0.66, \mathrm{p}<0.001$; C9orf72: $\mathrm{r}=0.75$, $\mathrm{p}<0.001 ; M A P T: \mathrm{r}=0.41, \mathrm{p}=0.017)$ and symptomatic mutation
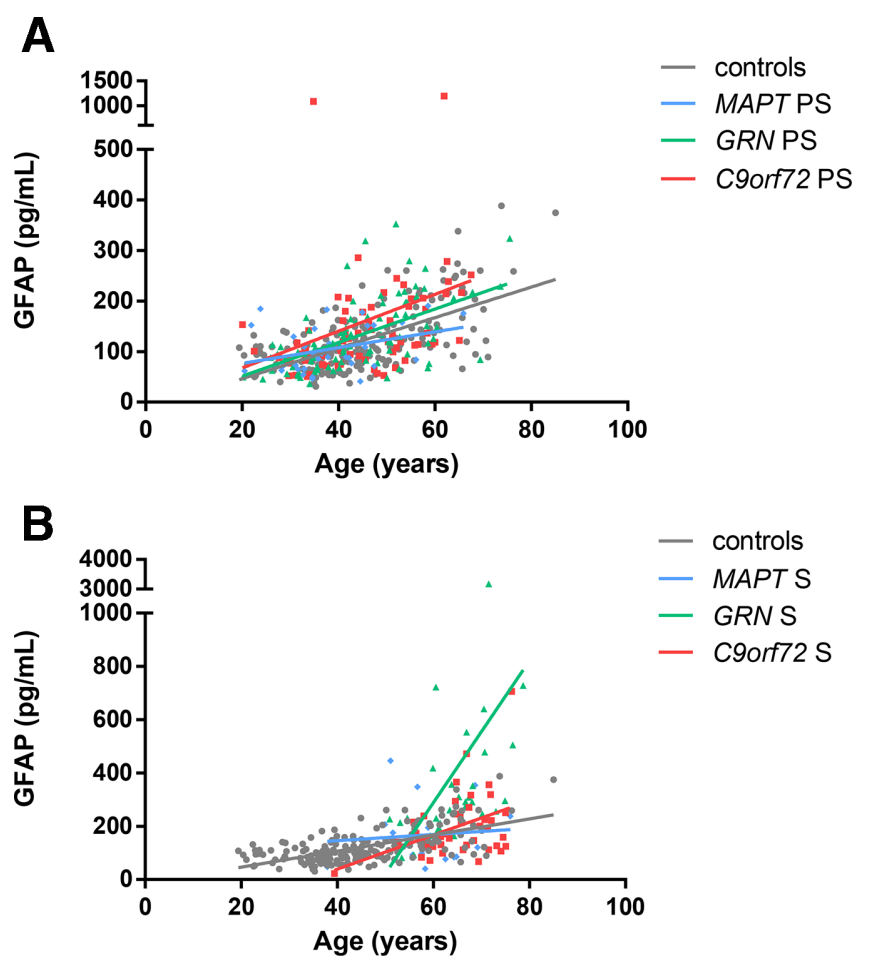

Figure 2 Correlation between plasma glial fibrillary acidic protein (GFAP) concentrations (pg/mL) and age: (A) presymptomatic and (B) symptomatic mutation carriers. carriers (GRN: r=0.38, $\mathrm{p}=0.036 ;$ C9orf72: $\mathrm{r}=0.57, \mathrm{p}<0.001$; $M A P T: \mathrm{r}=0.76, \mathrm{p}<0.001)$.

\section{Correlation with cognitive measures}

A significant negative correlation between GFAP concentrations and MMSE was seen in the presymptomatic GRN $(\mathrm{r}=-0.24$, $\mathrm{p}=0.033)$ and C9orf72 $(\mathrm{r}=-0.40, \mathrm{p}<0.001)$ but not MAPT $(\mathrm{r}=0.05, \mathrm{p}=0.801)$ mutation carriers. No significant correlation was seen during the symptomatic period in any of the genetic groups $(G R N$ : $\mathrm{r}=-0.29, \mathrm{p}=0.153$; C9orf72: $\mathrm{r}=-0.24$, $\mathrm{p}=0.146 ;$ MAPT: $\mathrm{r}=-0.48, \mathrm{p}=0.080)$.

No significant correlations were seen between GFAP concentration and FTLD-CDR sum of boxes score in either the presymptomatic or symptomatic period in any group: GRN: $\mathrm{r}=-0.04, \mathrm{p}=0.768$ presymptomatic, $\mathrm{r}=0.18, \mathrm{p}=0.409 \mathrm{symp}-$ tomatic; C9orf72: $\mathrm{r}=-0.17, \mathrm{p}=0.234$ presymptomatic, $\mathrm{r}=0.21$, $\mathrm{p}=0.321$ symptomatic; MAPT: $\mathrm{r}=-0.17, \mathrm{p}=0.446$ presymptomatic, $\mathrm{r}=0.14, \mathrm{p}=0.736$ symptomatic.

Correlation with cross-sectional imaging data

A significant negative correlation was seen between GFAP concentrations and both GRN and C9orf72 presymptomatic carrier brain volumes for frontal cortex $(\mathrm{r}=-0.23, \mathrm{p}=0.039$; $\mathrm{r}=-0.35, \mathrm{p}=0.002)$, temporal cortex $(\mathrm{r}=-0.35, \mathrm{p}=0.001$; $r=-0.27, p=0.024)$, cingulate cortex $(r=-0.24, p=0.027$; $\mathrm{r}=-0.44, \mathrm{p}<0.001)$ and insular cortex $(\mathrm{r}=-0.27, \mathrm{p}=0.016$; $r=-0.26, p=0.029)$ as well as whole brain $(r=-0.45, p<0.001)$ and parietal cortex $(\mathrm{r}=-0.33, \mathrm{p}=0.005)$ for the C9orf72 group (online supplementary table 3 ). No significant correlations were seen in the presymptomatic MAPT mutation carrier group or any of the symptomatic genetic groups.

\section{Correlation with longitudinal imaging data}

No significant positive correlation of GFAP concentration with longitudinal rates of atrophy were seen in any of the groups except for in the temporal cortex of symptomatic GRN mutation carriers $(\mathrm{r}=0.66, \mathrm{p}=0.010)$ (online supplementary table 4). However, within the same symptomatic GRN group there was also a trend in relationship between GFAP concentration and atrophy rates in the cingulate cortex $(r=0.55, \mathrm{p}=0.052)$.

\section{DISCUSSION}

In this study, we found that plasma GFAP concentration was significantly increased in genetic FTD but only in GRN mutation carriers, and not in those with C9orf72 expansions or mutations 
Table 3 Adjusted mean differences in plasma NfL concentrations between groups with $95 \%$ bias-corrected bootstrap CI

\begin{tabular}{|c|c|c|c|c|c|c|}
\hline $\mathrm{NfL}$ & C9orf72 PS & C9orf72 S & GRN PS & GRN S & MAPT PS & MAPT S \\
\hline Controls & $\begin{array}{l}9.0^{*} \\
(1.3 \text { to } 26.8)\end{array}$ & $\begin{array}{l}29.7 * * \\
\text { (18.7 to } 47.5)\end{array}$ & $\begin{array}{l}0.0 \\
(-3.1 \text { to } 3.3)\end{array}$ & $\begin{array}{l}70.5^{* *} \\
\text { (51.6 to } 92.6)\end{array}$ & $\begin{array}{l}0.9 \\
(-2.2 \text { to } 6.4)\end{array}$ & $\begin{array}{l}12.6^{*} \\
\text { (3.3 to } 26.1 \text { ) }\end{array}$ \\
\hline C9orf72 PS & & $\begin{array}{l}20.7^{*} \\
\text { (3.2 to } 36.1)\end{array}$ & $\begin{array}{l}-9.0^{*} \\
(-27.5 \text { to }-1.4)\end{array}$ & $\begin{array}{l}61.4^{* *} \\
(37.7 \text { to } 82.7)\end{array}$ & $\begin{array}{l}-8.1^{*} \\
(-27.0 \text { to }-0.6)\end{array}$ & $\begin{array}{l}3.6 \\
(-15.6 \text { to } 17.0)\end{array}$ \\
\hline C9orf72 S & & & $\begin{array}{l}-29.8^{* *} \\
(-47.7 \text { to }-18.7)\end{array}$ & $\begin{array}{l}40.7^{* *} \\
(16.5 \text { to } 62.2)\end{array}$ & $\begin{array}{l}-28.9 * * \\
(-46.2 \text { to }-16.9)\end{array}$ & $\begin{array}{l}-17.2^{*} \\
(-35.7 \text { to }-2.9)\end{array}$ \\
\hline GRN PS & & & & $\begin{array}{l}70.5^{* *} \\
(52.5 \text { to } 92.2)\end{array}$ & $\begin{array}{l}-0.9 \\
(-5.9 \text { to } 2.9)\end{array}$ & $\begin{array}{l}12.6^{*} \\
\text { (2.9 to } 25.2 \text { ) }\end{array}$ \\
\hline GRN S & & & & & $\begin{array}{l}-69.6^{* *} \\
(-91.7 \text { to }-50.6)\end{array}$ & $\begin{array}{l}-57.9^{* *} \\
(-81.5 \text { to }-36.8)\end{array}$ \\
\hline MAPT PS & & & & & & $\begin{array}{l}11.7^{*} \\
\text { (0.8 to 23.4) }\end{array}$ \\
\hline
\end{tabular}

Significant differences in bold: ${ }^{*} \mathrm{p}<0.05 ;{ }^{* *} \mathrm{p}<0.01$

$\mathrm{NfL}$, neurofilament light chain; PS, presymptomatic; S, symptomatic.

in the MAPT gene. In the presymptomatic period, higher concentrations were correlated with a lower cognitive score (MMSE) and lower brain volumes (in regions characteristically affected in FTD), potentially suggesting GFAP is increased in the late presymptomatic period. In the symptomatic period, higher concentrations were associated with faster rates of atrophy, suggesting GFAP levels are associated with disease intensity, and therefore progression and survival.

GFAP is a major constituent of the astrocytic cytoskeleton and its expression pattern is highly brain-enriched. ${ }^{27}$ Its levels increase following acute damage to astrocytes such as after a stroke $^{28}$ or traumatic brain injury, ${ }^{29}$ but also in relation to more chronic insults, such as in neurodegeneration, when astrocytes become reactive, increasing in size and proliferating, a process called astrogliosis. ${ }^{27}$ In neurodegeneration, increased GFAP concentrations in biofluid have been reported in Alzheimer's disease $^{30}$ (in both CSF $^{1719}$ and serum ${ }^{19}$ ) and amyotrophic lateral sclerosis ${ }^{18}$. Previous studies in FTD have found increased CSF concentrations in symptomatic patients within combined clinical $^{17}{ }^{19}$ and genetic cohorts ${ }^{18}$ but have not previously found changes in blood, ${ }^{19}$ nor investigated individual genetic groups previously. Our results suggest that there are differential increases within FTD, with concentrations being higher in people with $G R N$ mutations than in other groups. GRN encodes the progranulin protein, which is a secreted growth factor and known to be involved in many biological processes including inflammation, wound healing and cell proliferation. ${ }^{31} 32$ However, progranulin is also taken up by astrocytes for storage or transportation to the lysosomal compartment, ${ }^{33-35}$ and studies of GRN-deficient mice have shown the presence of astrogliosis. ${ }^{35-37}$ In vitro, progranulin seems to have a role of inactivating astrocytes with evidence that progranulin attenuates a pro-inflammatory phenotype of astrocytes. ${ }^{35}$ This suggests that deficiency of progranulin in GRN mutation carriers may lead to activation of pro-inflammatory phenotypes of astrocytes and subsequent astrogliosis, with increased levels of GFAP expression. People with GRN mutations have evidence of astrogliosis pathologically, including within areas of white matter damage ${ }^{38}$ (visible in a proportion of people in vivo as white matter hyperintensities on MR imaging, which have been previously shown to be unique to GRN mutations within familial FTD). ${ }^{39}$ Such damage increases as the neurodegeneration progresses, ${ }^{39}$ consistent with the pattern of increased plasma GFAP in our study. In contrast, levels were not increased in plasma in individuals with C9orf72 expansions or MAPT mutations. While astrogliosis is seen in animal models and at postmortem in both C9orf72-related ${ }^{40}$ and MAPT-related ${ }^{41} 42$ FTD, this may well be a late feature of the disease, or the extent of astrogliosis may be less. Future work will be required to investigate this further.

It is well established that multiple biomarkers of neurodegeneration increase in concentration with age, attributed to the reduction of neural integrity in the ageing brain. ${ }^{43}$ CSF GFAP concentrations have previously been shown to increase as one gets older, ${ }^{44}$ with multiple studies showing proliferation of astrocytes, increased GFAP immunoreactivity and elevated levels of GFAP mRNA with age. ${ }^{45-49}$ Consistent with this, we also found a significant positive correlation of plasma GFAP concentration with age in the majority of the groups. This highlights the importance of adjusting plasma GFAP concentrations for age in statistical analyses: symptomatic mutation carriers in the C9orf72 and $M A P T$ groups (as well as the GRN group) in this study showed increased levels of plasma GFAP compared with controls and their presymptomatic counterparts, but significance was lost once adjusting for age.

NfL, part of the axonal cytoskeleton, is released following cellular damage. A previous study has shown that NfL concentrations are increased in both the CSF and blood of symptomatic genetic FTD in all three mutation groups, C9orf72, GRN and MAPT. ${ }^{3}$ The results in this study replicate these findings in plasma, although we also found elevated levels in presymptomatic C9orf72 mutation carriers. In this latter group, NfL concentrations correlated negatively with MMSE $(r=-0.33, p=0.004$ : online supplementary table 5) and with brain volumes (whole brain, $\mathrm{r}=-0.53, \mathrm{p}<0.001$ and cortical regions: frontal, -0.51 , $<0.001$; temporal, $-0.37,0.001$; parietal, $-0.51,<0.001$; occipital, $-0.33,0.005$; cingulate, $-0.49,<0.001$; insula, $-0.47,<0.001$ : online supplementary tables 6 and 7 ), suggesting that NfL increases particularly towards the end of the presymptomatic period with increasing neurodegeneration. Although GRN NfL concentration was not significantly increased presymptomatically, a similar pattern of negative correlation with brain volumes was seen in this group (in whole brain and all cortical regions except the occipital lobe, $\mathrm{r}=-0.30$ to $-0.48, \mathrm{p} \leq 0.006$ ). $\mathrm{NfL}$ and GFAP concentrations were significantly correlated in all groups including controls, although the correlation coefficient varied from 0.38 to 0.76 . A similar correlation has been shown in CSF previously. ${ }^{44}$ As both increase with age (online supplementary table 8 for NfL correlations with age), the correlation is not unexpected, but other unexplained factors are likely to affect the different patterns within genetic FTD; interestingly, 
the lowest correlation $(\mathrm{r}=0.38)$ was in the symptomatic $G R N$ mutation carriers, suggesting that in this group astrogliosis and neurodegeneration are not so closely related.

Correlation of GFAP concentration with cognitive and imaging measures revealed a negative correlation, that is, higher concentration with a lower cognitive score and lower crosssectional brain volumes in FTD-related regions in presymptomatic GRN mutation carriers. This suggests that GFAP levels start to increase as the brain starts to decrease in volume, and as cognition starts to become affected thus in the later stages of the presymptomatic period in proximity to symptom onset. This would be an important biomarker for GRN-related FTD, as an increase in concentration from baseline during the presymptomatic period would identify a time around the onset of neurodegeneration, and potentially a time when therapeutic intervention may be optimal. Despite the lack of a significant increase in concentration in C9orf72 mutation carriers, a similar pattern of negative correlation with cognition and brain imaging was seen in the presymptomatic period-it would be useful in future studies to investigate the subset of C9orf72 expansion carriers that have increased GFAP concentrations, and how they differ from those with a lower concentration. In particular, it would be helpful to compare carriers with and without concomitant ALS. We also assessed whether GFAP correlated with the rate of brain atrophy measured with longitudinal brain imaging and found a significant positive correlation only in the symptomatic GRN carriers (in the temporal lobe), implicating an association of GFAP levels with the intensity of the disease process, that is, how fast the disease is progressing. With longitudinal follow-up of participants, it would therefore be hypothesised that higher GFAP concentration would be associated with shorter survival in GRN-related FTD.

While the multicentre nature of the GENFI study allows collection of samples from a large genetic cohort of FTD worldwide, there remains a relatively small number of cases in each group (leading to low statistical power to detect differences), particularly in the symptomatic carriers, and replication in a larger dataset would be helpful. Due to the nature of the disease process, the mean age of the controls overall is lower compared with the symptomatic mutation carriers, but nonetheless the same results are found whether performing an age-adjusted comparison (as presented above in Plasma GFAP concentration) or when symptomatic mutation carriers are compared with an age-matched and gender-matched subset of older controls (see online supplementary figure 2). The advantage of studying levels in plasma is that blood is more easily accessible and a relatively cost-efficient way to access bodily fluids in comparison to performing a lumbar puncture; in this study, the use of the ultrasensitive SIMOA assay allowed detection at a level in blood that other assays do not. However, it will be important to study CSF levels in more detail in this group, as concentrations can differ between blood and CSF. ${ }^{18}$ Lastly, despite significant differences between the groups, there is a substantial overlap in concentrations between carriers and controls: longitudinal study of GFAP concentration over time, particularly in participants that convert from presymptomatic to symptomatic status, will therefore be important to truly evaluate whether changes do occur towards the end of the presymptomatic period and how levels change with progression of disease.

In summary, plasma GFAP levels appear to be uniquely increased in GRN mutation carriers in the current study, and importantly, concentrations may well be abnormal during the late presymptomatic period, suggesting that GFAP might act as marker of proximity to symptom onset.

\section{Author affiliations}

${ }^{1}$ UK Dementia Research Institute, Department of Neurodegenerative Disease, University College London, London, UK

${ }^{2}$ Dementia Research Centre, UCL Queen Square Institute of Neurology, London, UK ${ }^{3}$ Centre for Medical Image Computing, University College London, London, UK ${ }^{4}$ Neuradiological Academic Unit, UCL Queen Square Institute of Neurology, London, UK

${ }^{5}$ Department of Neurology, Erasmus Medical Centre, Rotterdam, The Netherlands

${ }^{6}$ Cognitive Disorders Unit, Department of Neurology, Donostia University Hospital, San Sebastian, País Vasco, Spain

${ }^{7}$ Alzheimer's Disease and Other Cognitive Disorders Unit, Neurology Service, Hospital Clínic, Barcelona, Spain

${ }^{8}$ Centre for Neurodegenerative Disorders, Neurology Unit, Department of Clinical and Experimental Sciences, University of Brescia, Brescia, Italy

${ }^{9}$ Clinique Interdisciplinaire de Mémoire du CHU de Québec, Département des Sciences Neurologiques, Université Laval, Québec, Québec, Canada

${ }^{10}$ Sunnybrook Health Sciences Centre, Sunnybrook Research Institute, University of Toronto, Toronto, Ontario, Canada

${ }^{11}$ Tanz Centre for Research in Neurodegenerative Disease, University of Toronto, Toronto, Ontario, Canada

${ }^{12}$ Department of Geriatric Medicine, Karolinska University Hospital-Huddinge, Stockholm, Sweden

${ }^{13}$ Department of Biomedical, Surgical and Dental Sciences, University of Milan,

Centro Dino Ferrari, Milan, Italy

${ }^{14}$ Fondazione IRCCS Ca' Granda, Ospedale Maggiore Policlinico, Milan, Italy ${ }^{15}$ Department of Clinical Neurosciences, University of Cambridge, Cambridge, UK ${ }^{16}$ Department of Clinical Neurological Sciences, University of Western Ontario, London, Ontario, Canada

${ }^{17}$ Department of Neurodegenerative Diseases, Hertie Institute for Clinical Brain Research, Tübingen, Germany

${ }^{18}$ German Center for Neurodegenerative Diseases (DZNE), University of Tübingen, Tübingen, Germany

${ }^{19}$ Laboratory for Cognitive Neurology, Department of Neurosciences, KU Leuven, Leuven, Belgium

${ }^{20}$ Faculty of Medicine, University of Lisbon, Lisbon, Portugal

${ }^{21}$ Fondazione Istituto di Ricovero e Cura a Carattere Scientifico, Istituto Neurologico Carlo Besta, Milan, Italy

${ }^{22}$ Faculty of Medicine, University of Coimbra, Coimbra, Portugal

${ }^{23}$ Department of Neurology and Neurosurgery, McGill University, Montreal, Québec, Canada

${ }^{24}$ Department of Clinical Neurology, University of Oxford, Oxford, UK

${ }^{25}$ Faculty of Medical and Human Sciences, Institute of Brain, Behaviour and Mental Health, University of Manchester, Manchester, UK

${ }^{26}$ German Center for Neurodegenerative Diseases (DZNE), Munich, Germany

${ }^{27}$ Department of Neurology, Ludwig-Maximilians-University, Munich, Germany

${ }^{28}$ Munich Cluster for Systems Neurology (SyNergy), Munich, Germany

${ }^{29}$ IRCCS San Giovanni di Dio Fatebenefratelli, Brescia, Italy

${ }^{30}$ Department of Neuroscience, Psychology, Drug Research, and Child Health, University of Florence, Florence, Italy

${ }^{31}$ Department of Neurology, University of Ulm, Ulm, Germany

${ }^{32}$ Department of Psychiatry and Neurochemistry, Sahlgrenska Academy at the University of Gothenburg, Mölndal, Sweden

Twitter Martha S Foiani @marthafoiani, David Thomas @dave_mri and Simon Ducharme @sducharme66

Acknowledgements The authors would like to thank the members of the kindreds who have been active participants in the GENFI study.

Collaborators Martin N Rossor, Jason D Warren (Dementia Research Centre, Department of Neurodegenerative Disease, UCL Queen Square Institute of Neurology, London, UK); Nick C Fox (Dementia Research Centre, Department of Neurodegenerative Disease, UCL Queen Square Institute of Neurology, London, UK; Dementia Research Institute, Department of Neurodegenerative Disease, UCL Institute of Neurology, Queen Square, London, UK); Rita Guerreiro, Jose Bras (Center for Neurodegenerative Science, Van Andel Research Institute, Grand Rapids, Michigan, USA); Jennifer Nicholas (Department of Medical Statistics, London School of Hygiene and Tropical Medicine, London, UK); Simon Mead (MRC Prion Unit, Department of Neurodegenerative Disease, UCL Institute of Neurology, Queen Square, London, UK); Lize Jiskoot, Lieke Meeter, Jessica Panman, Janne Papma (Department of Neurology, Erasmus Medical Centre, Rotterdam, Netherlands); Rick van Minkelen (Department of Clinical Genetics, Erasmus Medical Centre, Rotterdam, Netherlands); Yolanda Pijnenburg (Amsterdam University Medical Centre, Amsterdam VUmc, Amsterdam, Netherlands); Myriam Barandiaran, Begoña Indakoetxea (Cognitive Disorders Unit, Department of Neurology, Donostia University Hospital, San Sebastian, Gipuzkoa, Spain; Neuroscience Area, Biodonostia Health Research Institute, San Sebastian, Gipuzkoa, Spain); Alazne Gabilondo, Mikel Tainta, Maria de Arriba, Ana Gorostidi, Miren Zulaica (Neuroscience Area, Biodonostia Health 
Research Institute, San Sebastian, Gipuzkoa, Spain); Jorge Villanua (OSATEK, University of Donostia, San Sebastian, Gipuzkoa, Spain); Zigor Diaz (1CITA Alzheimer, San Sebastian, Gipuzkoa, Spain); Sergi Borrego-Ecija, Jaume Olives, Albert Lladó, Mircea Balasa, Anna Antonell (Alzheimer's disease and Other Cognitive Disorders Unit, Neurology Service, Hospital Clínic, Barcelona, Spain); Nuria Bargallo (Imaging Diagnostic Center, Hospital Clínic, Barcelona, Spain), Enrico Premi, Maura Cosseddu Stefano Gazzina, Alessandro Padovani (Centre for Neurodegenerative Disorders, Neurology Unit, Department of Clinical and Experimental Sciences, University of Brescia, Brescia, Italy); Roberto Gasparotti (Neuroradiology Unit, University of Brescia, Brescia, Italy); Silvana Archetti (Biotechnology Laboratory, Department of Diagnostics, Spedali Civili Hospital, Brescia, Italy), Sandra Black, Sara Mitchell (Sunnybrook Health Sciences Centre, Sunnybrook Research Institute, University of Toronto, Toronto, Canada); Ekaterina Rogaeva (Tanz Centre for Research in Neurodegenerative Diseases, University of Toronto, Toronto, Canada); Morris Freedman (Baycrest Health Sciences, Rotman Research Institute, University of Toronto, Toronto, Canada), Ron Keren (The University Health Network, Toronto Rehabilitation Institute, Toronto, Canada); David Tang-Wai (The University Health Network, Krembil Research Institute, Toronto, Canada); Linn Öijerstedt (Department of Geriatric Medicine, Karolinska University Hospital-Huddinge, Stockholm, Sweden); Christin Andersson (Department of Clinical Neuroscience, Karolinska Institutet, Stockholm, Sweden); Vesna Jelic (Division of Clinical Geriatrics, Karolinska Institutet, Stockholm, Sweden); Hakan Thonberg (Center for Alzheimer Research, Divison of Neurogeriatrics, Karolinska Institutet, Stockholm, Sweden); Andrea Arighi, Chiara Fenoglio, Elio Scarpini (Fondazione IRCCS Ca' Granda Ospedale Maggiore Policlinico, Neurodegenerative Diseases Unit, Milan, Italy; University of Milan, Centro Dino Ferrari, Milan, Italy); Giorgio Fumagalli (Fondazione IRCCS Ca' Granda Ospedale Maggiore Policlinico, Neurodegenerative Diseases Unit, Milan, Italy; University of Milan, Centro Dino Ferrari, Milan, Italy; Department of Neurosciences, Psychology, Drug Research and Child Health (NEUROFARBA), University of Florence, Florence, Italy); Thomas Cope, Carolyn Timberlake, Timothy Rittman (Department of Clinical Neurosciences, University of Cambridge, Cambridge, UK); Christen Shoesmith (Department of Clinical Neurological Sciences, University of Western Ontario, London, Ontario Canada); Robart Bartha (Department of Medical Biophysics, The University of Western Ontario, London, Ontario, Canada; Centre for Functional and Metabolic Mapping, Robarts Research Institute, The University of Western Ontario, London, Ontario, Canada); Rosa Rademakers (Department of Neuroscience, Mayo Clinic, Jacksonville, Florida, USA): Carlo Wilke (Department of Neurodegenerative Diseases, Hertie-Institute for Clinical Brain Research and Center of Neurology, University of Tübingen, Tübingen, Germany; Center for Neurodegenerative Diseases (DZNE), Tübingen, Germany), Hans-Otto Karnarth (Division of Neuropsychology, Hertie-Institute for Clinical Brain Research and Center of Neurology, University of Tübingen, Tübingen, Germany); Benjamin Bender (Department of Diagnostic and Interventional Neuroradiology, University of Tübingen, Tübingen, Germany), Rose Bruffaerts (Laboratory for Cognitive Neurology, Department of Neurosciences, KU Leuven, Leuven, Belgium); Philip Vandamme (Neurology Service, University Hospitals Leuven, Belgium, Laboratory for Neurobiology, VIB-KU Leuven Centre for Brain Research, Leuven, Belgium); Mathieu Vandenbulcke (Geriatric Psychiatry Service, University Hospitals Leuven, Belgium; Neuropsychiatry, Department of Neurosciences, KU Leuven, Leuven, Belgium); Catarina B Ferreira (Laboratory of Neurosciences, Institute of Molecular Medicine, Faculty of Medicine, University of Lisbon, Lisbon, Portugal); Gabriel Miltenberger (45Faculty of Medicine, University of Lisbon, Lisbon, Portugal); Carolina Maruta (Laboratory of Language Research, Centro de Estudos Egas Moniz, Faculty of Medicine, University of Lisbon, Lisbon, Portugal); Ana Verdelho (Department of Neurosciences and Mental Health, Centro Hospitalar Lisboa Norte - Hospital de Santa Maria \& Faculty of Medicine, University of Lisbon, Lisbon, Portugal); Sónia Afonso (Instituto Ciencias Nucleares Aplicadas a Saude, Universidade de Coimbra, Coimbra, Portugal); Ricardo Taipa (Neuropathology Unit and Department of Neurology, Centro Hospitalar do Porto - Hospital de Santo António, Oporto, Portugal); Paola Caroppo, Giuseppe Di Fede, Giorgio Giaccone, Sara Prioni, Veronica Redaelli, Giacomina Rossi, Pietro Tiraboschi (Fondazione IRCCS Istituto Neurologico Carlo Besta, Milano, Italy); Diana Duro, Maria Rosario Almeida, Miguel Castelo-Branco (Faculty of Medicine, University of Coimbra, Coimbra, Portugal), Maria João Leitão (Centre of Neurosciences and Cell biology, Universidade de Coimbra, Coimbra, Portugal), Miguel Tabuas-Pereira, Beatriz Santiago (Neurology Department, Centro Hospitalar e Universitario de Coimbra, Coimbra, Portugal); Serge Gauthier (Alzheimer Disease Research Unit, McGill Centre for Studies in Aging, Department of Neurology \& Neurosurgery, McGill University, Montreal, Québec, Canada), Pedro Rosa-Neto (Translational Neuroimaging Laboratory, McGill Centre for Studies in Aging, McGill University, Montreal, Québec, Canada), Michele Veldsman (Nuffield Department of Clinical Neurosciences, Medical Sciences Division, University of Oxford, Oxford, UK); Toby Flanagan (Faculty of Biology, Medicine and Health, Division of Neuroscience and Experimental Psychology, University of Manchester, Manchester, UK); Catharina Prix, Tobias Hoegen, Elisabeth Wlasich, Sandra Loosli, Sonja Schonecker (Neurologische Klinik, Ludwig-Maximilians-Universität München, Munich, Germany); Elisa Semler, Sarah Anderl-Straub (Department of Neurology, University of Ulm, Ulm); Luisa Benussi, Giuliano Binetti, Roberta Ghidoni, Michela Pievani (Instituto di Recovero e Cura a Carattere Scientifico Istituto Centro San Giovanni di Dio Fatebenefratelli, Brescia, Italy); Gemma Lombardi, Benedetta
Nacmias, Camilla Ferrari, Valentina Bessi (Department of Neuroscience, Psychology, Drug Research, and Child Health, University of Florence, Florence, Italy).

Contributors $\mathrm{CH}$ and JR contributed to the study design, acquisition, analysis and interpretation of the data as well as drafting and revising the manuscript. All other authors (MSF, AJH, HZ, KM, RC, MB, MN, DMC, DT, CVG, IOCW, RS, JCVS, FM, RS-V, $B B, R J I L, M M, M C T, C G, D G$, JBR, EF, MS, RV, AdeM, FT, IS, SD, CRB, AG, JL, AD, GF, SS, $\mathrm{MO}$ ) contributed to the acquisition of data and study coordination as well as helping to critically review and revise the manuscript.

Funding The GENFI study has been supported by the Medical Research Council UK (MR/M023664/1), the Italian Ministry of Health and the Canadian Institutes of Health Research as part of a Centres of Excellence in Neurodegeneration grant, as well as other individual funding to investigators. This work was supported by the NIHR Queen Square Dementia Biomedical Research Unit, the NIHR UCL/H Biomedical Research Centre and the Leonard Wolfson Experimental Neurology Centre (LWENC) Clinical Research Facility, Alzheimer's Research UK, the Brain Research Trust and the Wolfson Foundation as well as an Alzheimer's Society grant (AS-PG-16-007). The biomarker measurements were funded in part by the UK Dementia Research Institute at UCL and by a Wellcome Trust Multi-User Equipment Grant. KM has received funding from an Alzheimer's Society PhD studentship. IOCW is supported by a MRC Clinical Research Training Fellowship (MR/M018288/1). RS-V is supported by an Alzheimer's Research UK Clinical Research Training Fellowship (ARUK-CRF2017B-2). JCVS was supported by the Dioraphte Foundation grant 0902-03-00, the Association for Frontemporal Dementias Research Grant 2009, The Netherlands Organization for Scientific Research (NWO) grant HCMI 056-13-018, ZonMw Memorabel (Deltaplan Dementie, project number 733051 042), Alzheimer Nederland and the Bluefield project. CG received funding from JPND-Prefrontals VR Dnr 529-2014-7504, VR 2015-02926 and 2018-02754, the Swedish FTD Initiative-Schörling Foundation, Alzheimer Foundation, Brain Foundation and Stockholm County Council ALF. DG received support from the EU Joint ProgrammeNeurodegenerative Disease Research (JPND) and the Italian Ministry of Health (PreFrontALS) grant 733051042. RS-V has received funding from Fundació Marató de TV3, Spain (grant no. 20143810). FM received funding from the Tau Consortium and the Center for Networked Biomedical Research on Neurodegenerative Disease (CIBERNED). JBR has received funding from the Wellcome Trust (103838) and the National Institute for Health Research (NIHR) Cambridge Biomedical Research Centre. MO has received funding from BMBF (FTLDC). MM has received funding from a Canadian Institutes of Health Research operating grant and the Weston Brain Institute and Ontario Brain Institute. RV has received funding from the Mady Browaeys Fund for Research into Frontotemporal Dementia. EF has received funding from a CIHR grant \#327387. HZ is a Wallenberg Academy Fellow. JR is a MRC Clinician Scientist (MR/M008525/1) and has received funding from the NIHR Rare Diseases Translational Research Collaboration (BRC149/NS/MH), the Bluefield Project and the Association for Frontotemporal Degeneration.

Competing interests None declared.

Patient consent for publication Not required.

Ethics approval Local ethics committees at each site approved the study and all participants provided written informed consent at enrolment.

Provenance and peer review Not commissioned; externally peer reviewed.

Data availability statement Data are available on reasonable request. Some GENFI data is available on reasonable request through application to the GENFI Data Access Committee.

\section{ORCID iDs}

Carolin Heller http://orcid.org/0000-0002-1934-6162

Martha S Foiani http://orcid.org/0000-0003-4157-2606

Katrina Moore https://orcid.org/0000-0002-4458-8390

Rhian Convery http://orcid.org/0000-0002-9477-1812

Martina Bocchetta http://orcid.org/0000-0003-1814-5024

Mollie Neason https://orcid.org/0000-0001-9419-7171

David Thomas http://orcid.org/0000-0003-1491-1641

Caroline V Greaves https://orcid.org/0000-0002-6446-1960

Ione OC Woollacott https://orcid.org/0000-0003-1166-6417

Rachelle Shafei https://orcid.org/0000-0002-4760-8684

John C Van Swieten http://orcid.org/0000-0001-6278-6844

Barbara Borroni http://orcid.org/0000-0001-9340-9814

James B Rowe http://orcid.org/0000-0001-7216-8679

Elizabeth Finger http://orcid.org/0000-0003-4461-7427

Markus Otto http://orcid.org/0000-0002-6647-5944

Jonathan D Rohrer https://orcid.org/0000-0002-6155-8417

\section{REFERENCES}

1 Woollacott IOC, Rohrer JD. The clinical spectrum of sporadic and familial forms of frontotemporal dementia. J Neurochem 2016;138 Suppl 1:6-31. 
2 Rohrer JD, Nicholas JM, Cash DM, et al. Presymptomatic cognitive and neuroanatomical changes in genetic frontotemporal dementia in the genetic frontotemporal dementia initiative (GENFI) study: a cross-sectional analysis. Lancet Neurol 2015;14:253-62.

3 Meeter LH, Dopper EG, Jiskoot LC, et al. Neurofilament light chain: a biomarker for genetic frontotemporal dementia. Ann Clin Trans/ Neurol 2016;3:623-36.

4 Galimberti D, Fumagalli GG, Fenoglio C, et al. Progranulin plasma levels predict the presence of GRN mutations in asymptomatic subjects and do not correlate with brain atrophy: results from the GENFI study. Neurobiol Aging 2018;62:245. e9-e12.

5 Meeter LHH, Gendron TF, Sias AC, et al. Poly(GP), neurofilament and grey matter deficits in C9orf72 expansion carriers. Ann Clin Transl Neurol 2018;5:583-97.

6 Meeter LHH, Patzke H, Loewen G, et al. Progranulin levels in plasma and cerebrospinal fluid in granulin mutation carriers. Dement Geriatr Cogn Dis Extra 2016;6:330-40.

7 Gendron TF, Chew J, Stankowski JN, et al. Poly(GP) proteins are a useful pharmacodynamic marker for C9ORF72-associated amyotrophic lateral sclerosis. Sci Trans/ Med 2017. [Epub ahead of print: 29 Mar 2017].

8 Lehmer C, Oeckl P, Weishaupt JH, et al. Poly- GP in cerebrospinal fluid links C9orf72 -associated dipeptide repeat expression to the asymptomatic phase of ALS / FTD. EMBO Mol Med 2017;9:859-68.

9 Scherling CS, Hall T, Berisha F, et al. Cerebrospinal fluid neurofilament concentration reflects disease severity in frontotemporal degeneration. Ann Neurol 2014;75:116-26.

10 Skillbäck T, Mattsson N, Blennow K, et al. Cerebrospinal fluid neurofilament light concentration in motor neuron disease and frontotemporal dementia predicts survival. Amyotroph Lateral Scler Frontotemporal Degener 2017;18:397-403.

11 Lewczuk P, Ermann N, Andreasson U, et al. Plasma neurofilament light as a potential biomarker of neurodegeneration in Alzheimer's disease. Alzheimers Res Ther 2018;10.

12 Rohrer JD, Woollacott IOC, Dick KM, et al. Serum neurofilament light chain protein is a measure of disease intensity in frontotemporal dementia. Neurology 2016:87:1329-36.

13 Wilke C, Preische O, Deuschle C, et al. Neurofilament light chain in FTD is elevated not only in cerebrospinal fluid, but also in serum. J Neurol Neurosurg Psychiatry 2016;87:1270-2.

14 Skillbäck T, Farahmand B, Bartlett JW, et al. CSF neurofilament light differs in neurodegenerative diseases and predicts severity and survival. Neurology 2014;83:1945-53

15 Colangelo AM, Alberghina L, Papa M. Astrogliosis as a therapeutic target for neurodegenerative diseases. Neurosci Lett 2014;565:59-64.

16 Umoh ME, Dammer EB, Dai J, et al. A proteomic network approach across the ALS FTD disease spectrum resolves clinical phenotypes and genetic vulnerability in human brain. EMBO Mol Med 2018;10:48-62.

17 Ishiki A, Kamada M, Kawamura Y, et al. Glial fibrillar acidic protein in the cerebrospinal fluid of Alzheimer's disease, dementia with Lewy bodies, and frontotemporal lobar degeneration. J Neurochem 2016:136:258-61.

18 Oeckl P, Weydt P, Steinacker P, et al. Different neuroinflammatory profile in amyotrophic lateral sclerosis and frontotemporal dementia is linked to the clinical phase. J Neurol Neurosurg Psychiatry 2019;90:4-10.

19 Oeckl P, Halbgebauer S, Anderl-Straub S, et al. Glial Fibrillary Acidic Protein in Serum is Increased in Alzheimer's Disease and Correlates with Cognitive Impairment. JAD 2019;67:481-8.

20 Rascovsky K, Hodges JR, Knopman D, et al. Sensitivity of revised diagnostic criteria for the behavioural variant of frontotemporal dementia. Brain 2011;134:2456-77.

21 Gorno-Tempini ML, Hillis AE, Weintraub S, et al. Classification of primary progressive aphasia and its variants. Neurology 2011;76:1006-14.

22 Brooks BR, Miller RG, Swash M, et al. El Escorial revisited: revised criteria for the diagnosis of amyotrophic lateral sclerosis. Amyotroph Lateral Scler Other Motor Neuron Disord 2000:1:293-9.

23 Armstrong MJ, Litvan I, Lang AE, et al. Criteria for the diagnosis of corticobasal degeneration. Neurology 2013;80:496-503.

24 Höglinger GU, Respondek G, Stamelou M, et al. Clinical diagnosis of progressive supranuclear palsy: the movement disorder Society criteria. Mov Disord 2017;32:853-64.
25 Knopman DS, Kramer JH, Boeve BF, et al. Development of methodology for conducting clinical trials in frontotemporal lobar degeneration. Brain 2008;131:2957-68.

26 Cardoso MJ, Modat M, Wolz R, et al. Geodesic information flows: Spatially-Variant graphs and their application to segmentation and fusion. IEEE Trans Med Imaging 2015;34:1976-88

27 Liddelow SA, Barres BA. Reactive astrocytes: production, function, and therapeutic potential. Immunity 2017;46:957-67.

28 Dvorak F, Haberer I, Sitzer M, et al. Characterisation of the diagnostic window of serum glial fibrillary acidic protein for the differentiation of intracerebral haemorrhage and ischaemic stroke. Cerebrovasc Dis 2009;27:37-41.

29 Papa L, Silvestri S, Brophy GM, et al. Gfap out-performs S100 $\beta$ in detecting traumatic intracranial lesions on computed tomography in trauma patients with mild traumatic brain injury and those with extracranial lesions. J Neurotrauma 2014;31:1815-22.

30 Olsson B, Lautner R, Andreasson U, et al. CSF and blood biomarkers for the diagnosis of Alzheimer's disease: a systematic review and meta-analysis. Lancet Neurol 2016;15:673-84

31 Cenik B, Sephton CF, Kutluk Cenik B, et al. Progranulin: a proteolytically processed protein at the crossroads of inflammation and neurodegeneration. J Biol Chem 2012;287:32298-306.

32 Chitramuthu BP, Bennett HPJ, Bateman A. Progranulin: a new avenue towards the understanding and treatment of neurodegenerative disease. Brain 2017:140:3081-104.

33 Almeida S, Zhou L, Gao F-B. Progranulin, a glycoprotein deficient in frontotemporal dementia, is a novel substrate of several protein disulfide isomerase family proteins. PLoS One 2011;6:e26454

34 Menzel L, Kleber L, Friedrich C, et al. Progranulin protects against exaggerated axonal injury and astrogliosis following traumatic brain injury. Glia 2017;65:278-92.

35 Petkau TL, Hill A, Leavitt BR. Core neuropathological abnormalities in progranulindeficient mice are penetrant on multiple genetic backgrounds. Neuroscience 2016;315:175-95

36 Ghoshal N, Dearborn JT, Wozniak DF, et al. Core features of frontotemporal dementia recapitulated in progranulin knockout mice. Neurobiol Dis 2012;45:395-408.

37 Yin F, Banerjee R, Thomas B, et al. Exaggerated inflammation, impaired host defense, and neuropathology in progranulin-deficient mice. J Exp Med 2010;207:117-28.

38 Woollacott IOC, Bocchetta M, Sudre CH, et al. Pathological correlates of white matter hyperintensities in a case of progranulin mutation associated frontotemporal dementia. Neurocase 2018;24:166-74.

39 Sudre $\mathrm{CH}$, Bocchetta M, Cash D, et al. White matter hyperintensities are seen only in GRN mutation carriers in the GENFI cohort. Neuroimage Clin 2017:15:171-80.

40 Chew J, Gendron TF, Prudencio M, et al. Neurodegeneration. C9orf72 repeat expansions in mice cause TDP-43 pathology, neuronal loss, and behavioral deficits. Science 2015;348:1151-4.

41 Hallmann A-L, Araúzo-Bravo MJ, Mavrommatis L, et al. Astrocyte pathology in a human neural stem cell model of frontotemporal dementia caused by mutant tau protein. Sci Rep 2017;7.

42 Domoto-Reilly K, Davis MY, Keene CD, et al. Unusually long duration and delayed penetrance in a family with FTD and mutation in MAPT (V337M). Am J Med Genet B Neuropsychiatr Genet 2017:174:70-4.

43 Raz N, Rodrigue KM. Differential aging of the brain: patterns, cognitive correlates and modifiers. Neurosci Biobehav Rev 2006:30:730-48.

44 Vågberg $\mathrm{M}$, Norgren N, Dring $\mathrm{A}$, et al. Levels and age dependency of neurofilament light and glial fibrillary acidic protein in healthy individuals and their relation to the brain parenchymal fraction. PLoS One 2015;10:e0135886.

45 Hayakawa N, Kato H, Araki T. Age-related changes of astorocytes, oligodendrocytes and microglia in the mouse hippocampal CA1 sector. Mech Ageing Dev 2007;128:311-6

46 Cotrina ML, Nedergaard M. Astrocytes in the aging brain. J Neurosci Res 2002;67:1-10

47 Kohama SG, Goss JR, Finch CE, et al. Increases of glial fibrillary acidic protein in the aging female mouse brain. Neurobiol Aging 1995;16:59-67.

48 Goss JR, Finch CE, Morgan DG. Age-Related changes in glial fibrillary acidic protein mRNA in the mouse brain. Neurobiol Aging 1991;12:165-70.

49 Lynch AM, Murphy KJ, Deighan BF, et al. The impact of glial activation in the aging brain. Aging Dis 2010;1:262-78. 